\title{
High Guanidinium Permeability Reveals Dehydration-Dependent Ion Selectivity in the Plasmodial Surface Anion Channel
}

\author{
Abdullah A. B. Bokhari, Neida K. Mita-Mendoza, Alexandra Fuller, \\ Ajay D. Pillai, and Sanjay A. Desai \\ The Laboratory of Malaria and Vector Research, National Institute of Allergy and Infectious Diseases, \\ National Institutes of Health, Rockville, MD 20852, USA \\ Correspondence should be addressed to Sanjay A. Desai; sdesai@niaid.nih.gov
}

Received 22 May 2014; Revised 19 July 2014; Accepted 23 July 2014; Published 19 August 2014

Academic Editor: Yoshinori Marunaka

Copyright (C) 2014 Abdullah A. B. Bokhari et al. This is an open access article distributed under the Creative Commons Attribution License, which permits unrestricted use, distribution, and reproduction in any medium, provided the original work is properly cited.

\begin{abstract}
Malaria parasites grow within vertebrate erythrocytes and increase host cell permeability to access nutrients from plasma. This increase is mediated by the plasmodial surface anion channel (PSAC), an unusual ion channel linked to the conserved clag gene family. Although PSAC recognizes and transports a broad range of uncharged and charged solutes, it must efficiently exclude the small $\mathrm{Na}^{+}$ion to maintain infected cell osmotic stability. Here, we examine possible mechanisms for this remarkable solute selectivity. We identify guanidinium as an organic cation with high permeability into human erythrocytes infected with Plasmodium falciparum, but negligible uptake by uninfected cells. Transport characteristics and pharmacology indicate that this uptake is specifically mediated by PSAC. The rank order of organic and inorganic cation permeabilities suggests cation dehydration as the rate-limiting step in transport through the channel. The high guanidinium permeability of infected cells also allows rapid and stringent synchronization of parasite cultures, as required for molecular and cellular studies of this pathogen. These studies provide important insights into how nutrients and ions are transported via PSAC, an established target for antimalarial drug development.
\end{abstract}

\section{Introduction}

Malaria parasites are intracellular pathogens that invade, grow, and replicate asexually within erythrocytes; the clinical sequelae of malaria are, in large measure, determined by modification and eventual destruction of host erythrocytes. During its $\sim 48 \mathrm{~h}$ intracellular cycle, the human pathogen $P$. falciparum remodels its host cell by generating a membranous network in erythrocyte cytosol and altering erythrocyte membrane properties such as adhesiveness and permeability to various organic and inorganic solutes [1-4].

This increased permeability is mediated by the plasmodial surface anion channel (PSAC), identified by patch-clamp studies of the host cell membrane [5]. PSAC activity and the associated clag multigene family are conserved in all Plasmodium spp. [6-9], suggesting a function required for intracellular parasite survival. In vitro growth inhibition studies using PSAC inhibitors and modified media have implicated an essential role in parasite nutrient acquisition [10], with sugars, amino acids, purines, and some vitamins all having established uptake [11-13].

As a shared ion channel for these structurally divergent nutrients, PSAC has broad selectivity for solutes of varying charge and size $[11,14,15]$. At the same time, this channel must efficiently exclude $\mathrm{Na}^{+}$, an impermeant cation responsible for the osmotic stability of erythrocytes in plasma [16]. PSAC excludes $\mathrm{Na}^{+}$by $10^{3.5}$ - to $10^{5}$-fold when compared to $\mathrm{Cl}^{-}$, a remarkable feat for a channel that allows large organic cations to pass [14]. Consistent with fine-tuning of PSAC solute selectivity by evolutionary pressures, this level of $\mathrm{Na}^{+}$ exclusion is sufficient to prevent osmotic lysis of infected cells before completion of the parasite's intracellular cycle [17]. Although being low, $\mathrm{Na}^{+}$flux through this channel is sufficient to remodel the host erythrocyte's cation concentrations and affect parasite activities [17-19]. Structure-function 
studies with mammalian ion channels have revealed mechanisms for selecting a specific ion and excluding nearly all other solutes [20], but the reverse problem-broad permeability with exclusion of the small $\mathrm{Na}^{+}$ion by this parasite channel-requires distinct solutions that will have fundamental implications for understanding permeation.

Here, we examine possible mechanisms for PSAC's unusual selectivity and identify guanidinium $\left(\mathrm{Gdm}^{+}\right)$as a cation with high permeability. We show that monovalent cations have permeabilities that increase with cation ionic radius, contradicting pore sieving predictions and paralleling a similar relationship for anions in this channel. High $\mathrm{Gdm}^{+}$ permeability also enables stringent synchronization of parasite cultures, as commonly needed for molecular and cellular studies of malaria parasites. These findings suggest a testable model for how the channel achieves its unusual solute selectivity.

\section{Materials and Methods}

2.1. Parasite Cultivation and Synchronization. Human erythrocytes were obtained from anonymous donors (Interstate Blood Bank, Memphis, TN) and used for in vitro P. falciparum cultivation of indicated parasite lines in RPMI-1640 medium supplemented with $0.5 \%$ lipid-rich bovine albumin (MP Biomedicals, Solon, $\mathrm{OH}$ ); cultures were maintained at $37^{\circ} \mathrm{C}$ under $5 \% \mathrm{O}_{2}, 5 \% \mathrm{CO}_{2}$, and $90 \% \mathrm{~N}_{2}$.

To assess the efficiency of synchronization conditions, asynchronous parasite cultures were treated with either $300 \mathrm{mM}$ D-sorbitol or $150 \mathrm{mM}$ guanidinium chloride (Gdm$\mathrm{Cl}$ ) in a buffered solution (20 mM HEPES, $0.1 \mathrm{mg} / \mathrm{mL}$ BSA, pH 7.4 with $\mathrm{NaOH}$ ); each experiment included treatment with standard culture medium as a matched control. Synchronization involved 5 or $30 \mathrm{~min}$ incubations at room temperature and was terminated by addition of 10 volumes of culture medium. After centrifugation to remove the lysis solution, the cells were resuspended in culture medium and returned to $37^{\circ} \mathrm{C}$ for cultivation without additional washing. Parasite stage and growth were evaluated after $24 \mathrm{~h}$ using microscopic examination of Giemsa-stained smears.

2.2. Osmotic Lysis Transport Assays. Solute transport assays were performed as described previously [21]. Trophozoiteinfected erythrocytes were harvested and enriched using the percoll/sorbitol method, washed, and resuspended at $0.1 \%$ hematocrit in osmotic lysis solutions containing either $280 \mathrm{mM}$ sorbitol or $150 \mathrm{mM}$ Gdm-Cl buffered with $20 \mathrm{mM}$ HEPES, $0.1 \mathrm{mg} / \mathrm{mL}$ BSA, $\mathrm{pH}$ 7.4. The permeability of other cations was identically measured; each salt produced negligible hemolysis of uninfected cells (not shown). Where present, inhibitors were added from DMSO stock solutions. Solute transport was quantified by tracking transmittance of $700 \mathrm{~nm}$ light through a $1 \mathrm{~mL}$ cell suspension; kinetics were measured at indicated temperatures using a spectrophotometer (DU640 with Peltier temperature control, Beckman Coulter, Fullerton, CA). Inhibitor dose response experiments were normalized to matched controls without inhibitor; a normalized permeability at each inhibitor concentration $\left(P_{i}\right)$ was calculated according to $P_{i}=t_{o} / t_{i}$, where $t_{o}$ and $t_{i}$ correspond to the time required to reach a threshold level of lysis without and with inhibitor, respectively. This equation is based on a quantitative and inverse relationship between solute transport and time to cell lysis [21]. Permeability estimates and inhibitor affinities determined using this method match those obtained with tracer flux and patch-clamp $[5,7$, $10,21]$.

2.3. Sybr Green Measurements. Toxicity of $\mathrm{Gdm}-\mathrm{Cl}$ was evaluated using parasite cultures after synchronization with two consecutive $30 \mathrm{~min} \mathrm{D}$-sorbitol treatments. These synchronized cultures were treated with either $150 \mathrm{mM} \mathrm{Gdm}-\mathrm{Cl}$, $20 \mathrm{mM}$ HEPES, $0.1 \mathrm{mg} / \mathrm{mL}$ BSA, pH 7.4, or culture medium for $5 \mathrm{~min}$ at room temperature. After adding 10 volumes of culture medium, the cells were centrifuged to remove the medium and resuspended to $2 \%$ hematocrit in culture medium prior to plating in 96-well microplates. After cultivation for $72 \mathrm{~h}$, parasite DNA production was quantified with SYBR Green I nucleic acid stain as described previously [17].

\section{Results}

3.1. High PSAC Permeability to Guanidinium ${ }^{+}$. We sought to examine PSAC's unusual solute selectivity profile and recognized that blasticidin $S$ and leupeptin, toxins that reach their intracellular parasite targets via PSAC [22-24], are both bulky guanidine derivatives with molecular weights $>420 \mathrm{Da}$. Unsubstituted guanidine is positively charged at physiological $\mathrm{pH}$ and its conjugate acid, the guanidinium ion $\left(\mathrm{Gdm}^{+}\right.$, Figure 1(a) inset), has been used to study transport through other ion channels [25]. We therefore examined $\mathrm{Gdm}^{+}$permeability in infected erythrocytes and used a quantitative transmittance assay [26]. These studies revealed rapid osmotic lysis of infected cells in isotonic $\mathrm{Gdm}-\mathrm{Cl}$; the halftime, $0.89 \pm 0.08 \mathrm{~min}$, was significantly less than in isotonic sorbitol $\left(6.7 \pm 0.5 \mathrm{~min}, P<10^{-8}\right)$, a highly permeant sugar alcohol. Although net uptake of the Gdm-Cl salt depends on both $\mathrm{Gdm}^{+}$and $\mathrm{Cl}^{-}$permeabilities to maintain electroneutrality, conductive $\mathrm{Cl}^{-}$transport at this membrane is greater and not rate-limiting $[4,21]$. Because there is a quantitative and inverse relationship between osmotic lysis half-time and solute permeability [21], these measurements implicate 8-fold greater permeability for $\mathrm{Gdm}^{+}$than for sorbitol. In contrast, uninfected human erythrocytes exhibited low $\mathrm{Gdm}^{+}$permeability and were osmotically stable in $\mathrm{Gdm}-\mathrm{Cl}$ (bottom trace, Figure 1(a)).

The nonspecific PSAC inhibitor, furosemide, inhibited $\mathrm{Gdm}^{+}$uptake, suggesting channel-mediated transport (Figure 1(b)) [27]. Notably, the levels of inhibition achieved with $200 \mu \mathrm{M}$ and $2 \mathrm{mM}$ concentrations of furosemide resembled those for a subset of PSAC substrates that access two different mechanisms of transport through this channel [15, 28]. These two mechanisms exhibit differences in inhibitor efficacy: while the transport of some solutes is abolished by $200 \mu \mathrm{M}$ furosemide, other solutes, collectively referred to as "R+" solutes, exhibit significant residual uptake via PSAC that can be blocked by a higher furosemide concentration ( $2 \mathrm{mM})$. Remarkably, PSAC inhibitors from multiple chemical scaffolds exhibit a similar 10-fold reduction in potency when 


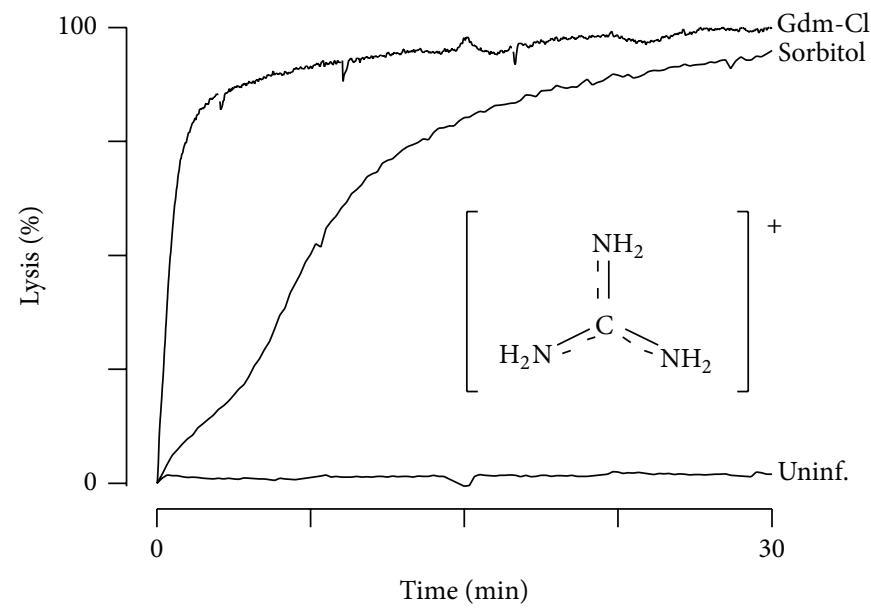

(a)

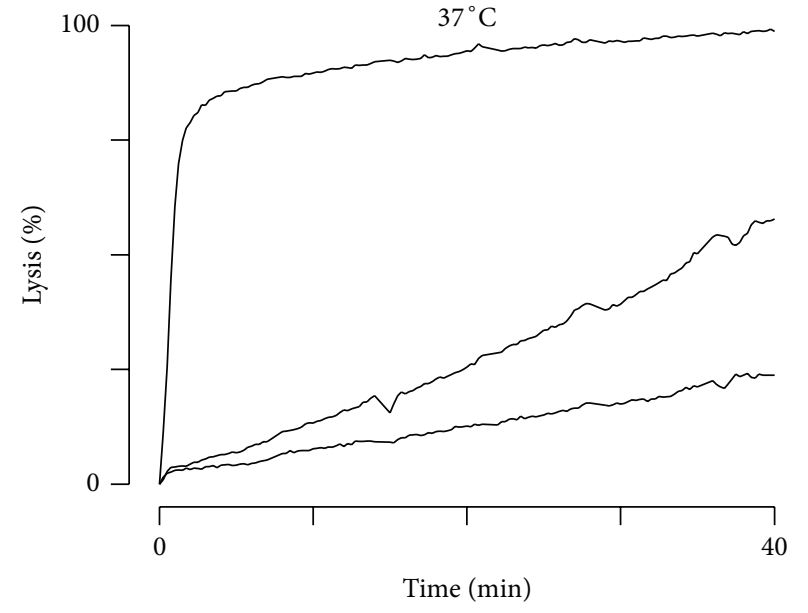

(b)

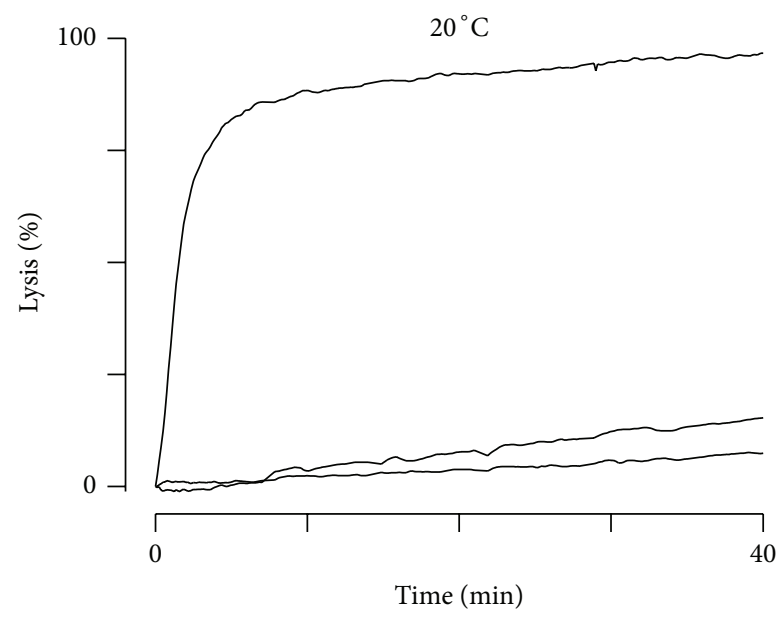

(c)

FIGURE 1: High $\mathrm{Gdm}^{+}$permeability in infected but not uninfected RBCs. (a) Osmotic lysis kinetics for infected erythrocytes in Gdm-Cl or sorbitol at $37^{\circ} \mathrm{C}$ (top two traces). Notice the faster kinetics in $\mathrm{Gdm}-\mathrm{Cl}$. Uninfected cells do not lyse in $\mathrm{Gdm}-\mathrm{Cl}$ (bottom trace). Inset shows the structure of $\mathrm{Gdm}^{+}$, which has a net +1 charge distributed amongst three primary amines. (b and c) Osmotic lysis kinetics for infected cells in $\mathrm{Gdm}-\mathrm{Cl}$ with 0,200 , or $2000 \mu \mathrm{M}$ furosemide (top to bottom traces, resp.). $200 \mu \mathrm{M}$ furosemide produces incomplete inhibition at $37^{\circ} \mathrm{C}$ but is more effective at $20^{\circ} \mathrm{C}$ (panels b and c, resp.), suggesting that $\mathrm{Gdm}^{+}$is an $\mathrm{R}+$ solute.

transport is examined with each $\mathrm{R}+$ solute. This observation suggests two distinct mechanisms used by this channel to recognize and transport solutes. Because the residual transport mechanism has steep temperature dependence [28], we examined $\mathrm{Gdm}^{+}$transport at $20^{\circ} \mathrm{C}$; under this condition, $200 \mu \mathrm{M}$ furosemide largely abolished uptake (Figure 1(c)), as reported for all other known $\mathrm{R}+$ solutes [15]. These experiments suggest $\mathrm{Gdm}^{+}$is transported via PSAC as an $\mathrm{R}+$ solute.

Because furosemide is nonspecific, we examined the mechanism of $\mathrm{Gdm}^{+}$uptake further with ISPA-28, a potent and specific small molecule inhibitor identified by highthroughput screening [7]. ISPA-28 blocks PSAC activity associated with the Dd2 parasite line $\left(K_{0.5}=56 \mathrm{nM}\right)$ but is largely inactive against channel activity induced by other parasite lines such as HB3 $\left(K_{0.5}=43 \mu \mathrm{M}\right)$; this compound's unique specificity enabled identification of clag3 genes and the channel's role in nutrient uptake through genetic mapping and DNA transfection experiments $[7,10]$. A short variable motif on the CLAG3 protein is exposed at the host cell surface and has been implicated in ISPA-28 binding [29].

ISPA-28 inhibited $\mathrm{Gdm}^{+}$uptake into cells infected with Dd2 but not those infected with HB3 parasites (Figures 2(a) and 2(b)). To explore whether other transporters contribute to $\mathrm{Gdm}^{+}$uptake after infection, we quantified ISPA-28 inhibition and compared block to that for sorbitol, a solute whose uptake via PSAC is well-established [7, 26]; these transport inhibition studies were performed at $15^{\circ} \mathrm{C}$ to reduce errors due to the residual transport mechanism described above. In both Dd2 and HB3 parasite lines, these dose response studies revealed quantitatively concordant inhibition of $\mathrm{Gdm}^{+}$and 


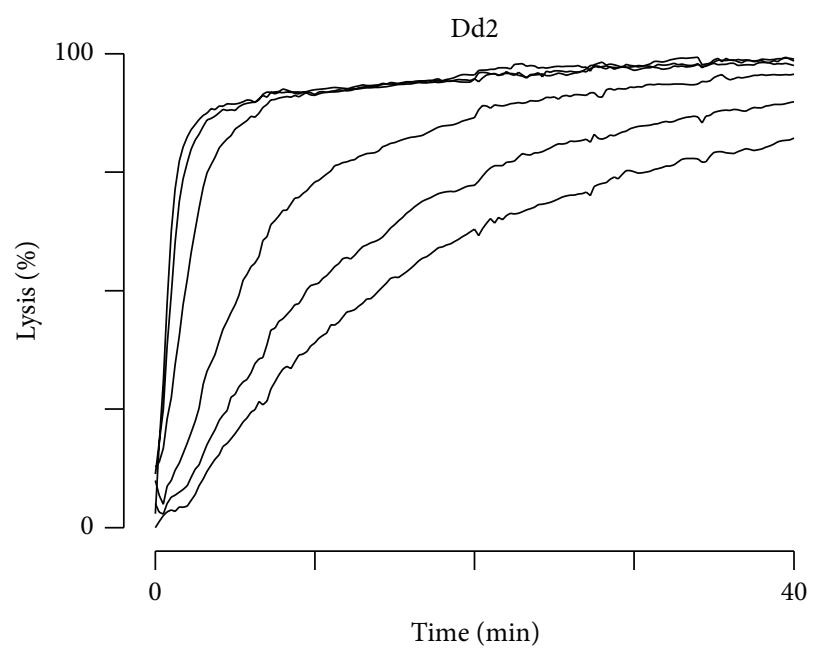

(a)

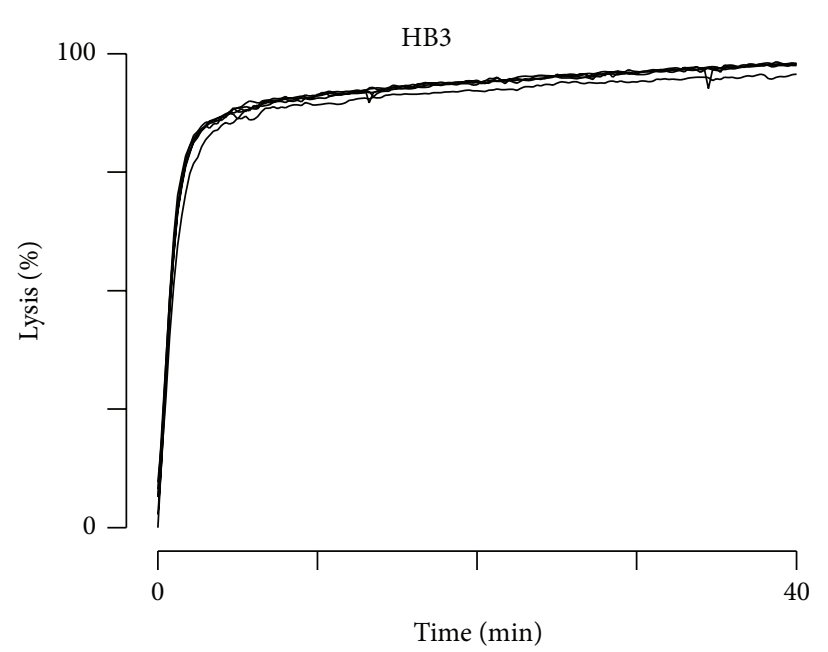

(b)

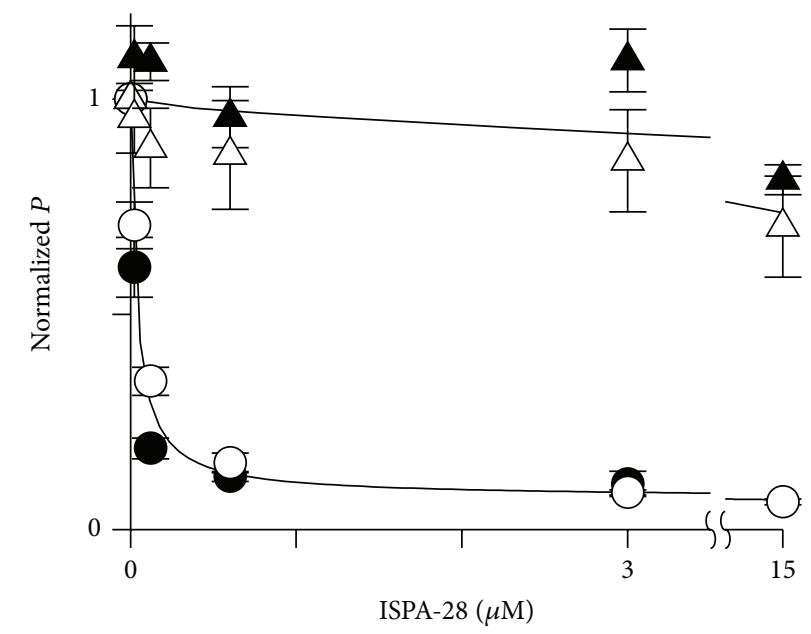

(c)

Figure 2: $\mathrm{Gdm}^{+}$uptake is primarily via PSAC. (a and b) Osmotic lysis kinetics for $\mathrm{Gdm}^{+}$uptake into Dd2 and $\mathrm{HB} 3$ at $15^{\circ} \mathrm{C}$. Traces reflect inhibition dose responses using $0,0.024,0.12,0.6,3.0$, and $15 \mu \mathrm{M}$ ISPA-28 (top to bottom, resp., in each panel). While inhibition is clear with Dd2-infected cells, there is negligible effect with HB3-infected cells. (c) Symbols represent mean \pm S.E.M. of tallied dose responses from experiments as in panels (a) and (b) for Gdm-Cl and sorbitol (white and black symbols, resp.) using Dd2- and HB3-infected cells (circles and triangles, resp.). The $\mathrm{Gdm}^{+}$and sorbitol dose responses do not differ $(n=3$ trials at each concentration, $P>0.1$ for comparisons between solutes in each parasite.).

sorbitol uptake by ISPA-28 (Figure 2(b)), indicating that $\mathrm{Gdm}^{+}$uptake is mediated primarily by PSAC.

3.2. Low Toxicity of $\mathrm{Gdm}^{+}$Permits Stringent Synchronization of Parasite Cultures. Sorbitol treatment, the current method of choice for synchronizing parasite cultures [30], is based on osmotic lysis of trophozoite-infected cells due to PSACmediated uptake [21]; it spares immature ring-infected cells, which lack this channel activity [31]. Two limitations include a requirement for relatively long incubations in sorbitol and poor stringency of synchronization. To achieve tighter synchrony for molecular studies such as stage-specific gene transcription, it is often necessary to use two or more rounds of sorbitol synchronization, making the procedure time- and effort-intensive. Alternative methods, such as gelatin floatation or enrichment of mature infected cells on magnetic columns $[32,33]$, have low yield or are also time-consuming.

We tested whether the greater permeability of $\mathrm{Gdm}^{+}$ allows improved synchronization by treating asynchronous cultures with either $\mathrm{Gdm}-\mathrm{Cl}$ or sorbitol. A $5 \mathrm{~min} \mathrm{Gdm}-\mathrm{Cl}$ treatment was more effective than either a 5 or 30 min sorbitol exposure, as quantified with examination of Giemsa-stained smears after subsequent cultivation for $24 \mathrm{~h}$ (Figure 3 (a), $P<$ 0.05). Although improved synchronization is presumably determined by the greater $\mathrm{Gdm}^{+}$permeability, the nearphysiological ionic strength of the $\mathrm{Gdm}-\mathrm{Cl}$ solution may also help; the lower ionic strength of sorbitol solutions may cause erythrocyte aggregation [34], leading to delayed solute uptake and osmotic lysis of some cells. 


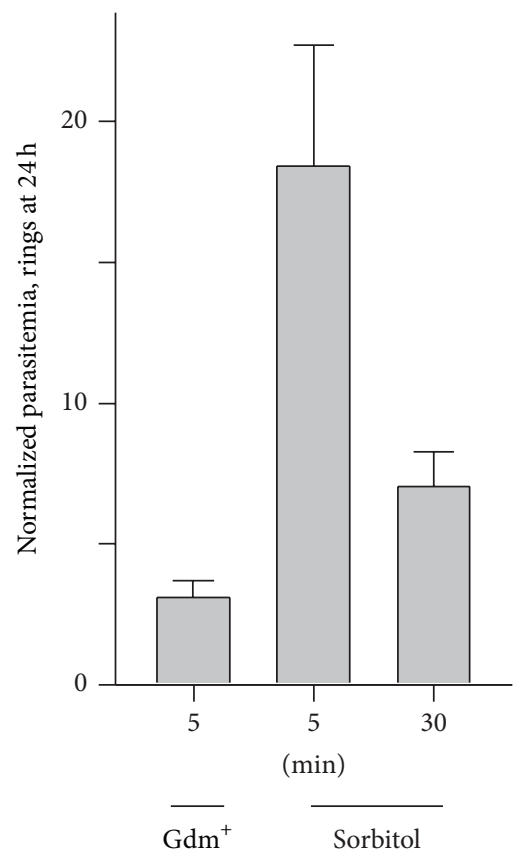

(a)

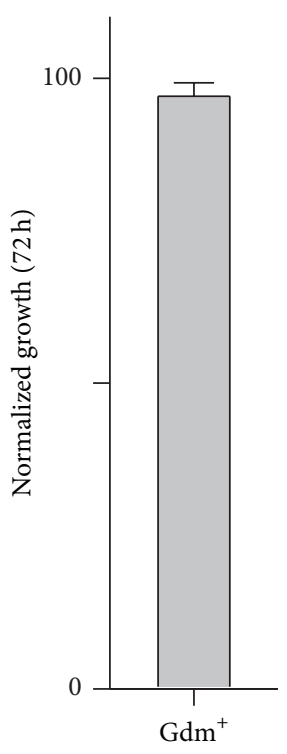

(b)

FIGURE 3: Stringent synchronization of cultures using Gdm-Cl. (a) Mature infected cells surviving synchronization with Gdm-Cl or sorbitol for indicated durations, as quantified using ring-stage parasitemia $24 \mathrm{~h}$ after treatment. A $5 \mathrm{~min} \mathrm{Gdm}$-Cl treatment is the most effective. (b) $72 \mathrm{~h}$ parasite growth after a $5 \mathrm{~min}$ treatment of synchronous cultures with $\mathrm{Gdm}-\mathrm{Cl}$, normalized to matched cultures sham-treated with culture medium. Bars represent mean \pm S.E.M. of 9 replicates from 3 experiments.

Because $\mathrm{Gdm}^{+}$is a strong protein denaturant at high concentrations, we wondered whether this synchronization strategy would be toxic to parasite cultures. We therefore treated ring-stage cultures with isotonic $\mathrm{Gdm}-\mathrm{Cl}$ solution and quantified subsequent parasite growth. Comparison to a sham treatment using standard culture medium revealed no change in parasite growth (Figure $3(\mathrm{~b}), P=0.36$ ), indicating that this treatment is not toxic to cultures. Experiments using a significantly longer $\mathrm{Gdm}^{+}$treatment of 30 min yielded measurable toxicity, but we did not detect accumulated toxicity with prolonged, regular use of $5 \mathrm{~min} \mathrm{Gdm}-\mathrm{Cl}$ treatments over consecutive asexual cycles (not shown).

Protein denaturation by $\mathrm{Gdm}-\mathrm{Cl}$ also cannot account for the apparent high $\mathrm{Gdm}^{+}$permeability via PSAC. Such models would predict hemolysis of uninfected erythrocytes in $\mathrm{Gdm}-\mathrm{Cl}$ solutions, which was not detected (Figure 1(a)). Denaturation would also not be consistent with block by ISPA-28, a highly specific PSAC inhibitor. Quantitatively concordant dose responses for inhibition of $\mathrm{Gdm}^{+}$and sorbitol uptake (Figure 2), when combined with insights from single channel patch-clamp using this inhibitor [7], implicate permeation through a channel pore not compromised by $\mathrm{Gdm}-\mathrm{Cl}$ exposure.

3.3. PSAC Permeabilities to Other Monovalent Cations. In contrast to $\mathrm{Gdm}^{+}$and various organic cations [14, 35], PSAC maintains a very low $\mathrm{Na}^{+}$permeability [16]. To explore possible mechanisms, we quantified the relative permeabilities of organic and inorganic cations with osmotic lysis kinetics

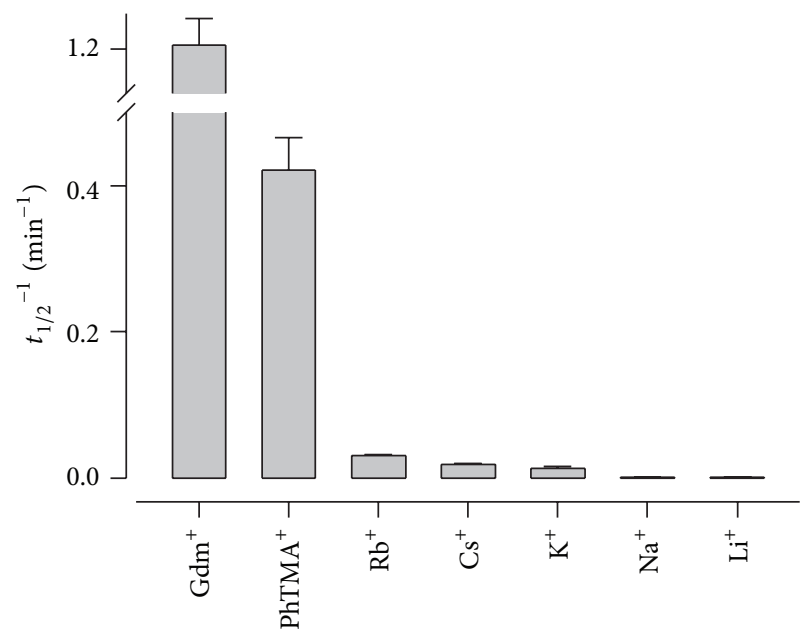

FIGURE 4: Dehydration-dependent permeation of cations. Mean \pm S.E.M. apparent permeability coefficients for indicated cations, determined as the reciprocal of the osmotic lysis halftime in buffered solutions of each chloride salt at $37^{\circ} \mathrm{C}$.

for infected cells in buffered solutions of each chloride salt (Figure 4). As expected, $\mathrm{Gdm}^{+}$was the most permeant of these cations. PhTMA ${ }^{+}$had substantial permeability that was 3-fold lower. $\mathrm{Cs}^{+}, \mathrm{Rb}^{+}$, and $\mathrm{K}^{+}$, large group $1 \mathrm{~A}$ alkali metals with ionic radii of $1.67,1.48$, and 1.33 Á, respectively, had more modest but still clearly resolved uptake. $\mathrm{Na}^{+}$and $\mathrm{Li}^{+}$, smaller group $1 \mathrm{~A}$ metals with radii of 0.98 and $0.68 \AA$, 
respectively, had negligible permeabilities. While anion fluxes through PSAC have been studied using both single-channel and whole-cell patch-clamp configurations, the significantly lower permeabilities of the cations in Figure 4 prohibit measurement of cation-specific currents with patch-clamp methods. These currents would be overwhelmed by the larger fluxes of anions such as $\mathrm{Cl}^{-}$, necessarily present at stoichiometric levels due to electroneutrality.

\section{Discussion}

The increased permeability of infected erythrocytes to small solutes is one of the earliest identified cellular phenotypes in malaria research [36]; studies from many groups have defined the range of permeant solutes and identified inhibitors $[3,14$, 37-39]. Although several distinct channels have been proposed for the infected cell membrane [40], recent chemical screens and molecular studies have implicated PSAC as a shared route for most solutes with increased permeability $[7,26]$. Parasite CLAG proteins, which lack homology to known channel proteins from other organisms, play a critical but incompletely understood role in formation of this channel $[8,10]$. Permeating solutes may be uncharged or zwitterionic or may carry a net positive or negative charge. Solutes up to $670 \mathrm{Da}$ in size have significant uptake [16], but the small $\mathrm{Na}^{+}$and $\mathrm{Li}^{+}$ions are effectively excluded (Figure 4). The combination of broad permeability to large solutes and effective exclusion of specific small ions is without precedent amongst other ion channels. This unusual selectivity profile appears to have been selected by evolutionary pressures that require uptake of diverse nutritive solutes, evasion of host immune responses, and a very low $\mathrm{Na}^{+}$permeability to prevent osmotic lysis of infected cells in the bloodstream, where $\mathrm{Na}^{+}$is the main osmotically active solute [41].

Here, we examined this unprecedented solute selectivity by quantifying cation transport through PSAC. We found that permeability increased with ionic size for group 1A cations (Figure 4), paralleling a similar relationship described for halide and pseudohalide anions identified through patchclamp, $\mathrm{SCN}^{-} \gg \mathrm{I}^{-}>\mathrm{Br}^{-}>\mathrm{Cl}^{-}$[4]. These findings contradict the predictions of simple pore sieving models, which expect the smallest solutes to have the greatest permeabilities. Instead, there appears to be a controlling effect of ion dehydration, the process of removing the shell of water molecules around dissolved ions $[42,43]$. For both cation and anion series, greater PSAC permeability correlates precisely with lower energy requirement for dehydration.

In particular, $\mathrm{Gdm}^{+}$and $\mathrm{SCN}^{-}$are notable as the cation and anion with the fastest transport rates. $\mathrm{Gdm}^{+}$is one of the most weakly hydrated ions known; it interacts poorly with water because a single positive charge is diffusely shared by three nitrogen atoms and because it has a rigid structure unable to interact well with water molecules [44]. $\mathrm{SCN}^{-}$also has a low charge density and is the least hydrated of all the anions in the Hofmeister series [45]. The poor hydration of these ions also accounts for their strong denaturant properties when present at higher concentrations [44].

Studies on $\mathrm{K}^{+}, \mathrm{Na}^{+}$, and $\mathrm{Ca}^{++}$channels suggest that permeating ions must be dehydrated to fit within the pore
$[20,46]$; dehydration is thought to allow specific interactions with the channel protein and enable selective transport. In this context, it is surprising that our studies implicate dehydration as a critical step in transport through PSAC: broad permeability to bulky organic solutes typically suggests a large pore capable of accommodating hydrated ions. We propose that ion dehydration may serve a distinct role in this channel's case by facilitating the selective exclusion of $\mathrm{Na}^{+}$. The energy required to dehydrate $\mathrm{Na}^{+}, 91.2 \mathrm{kcal} / \mathrm{mol}$ [46], is very large indeed. $\mathrm{Na}^{+}$channels compensate for this energy barrier by providing a strong binding site for $\mathrm{Na}^{+}$in the pore; in contrast, the PSAC pore offers negligible compensation, with an Eisenman selectivity sequence that corresponds to the weakest theoretical binding site for permeating ions. Under such conditions, large, easily dehydrated ions and nutritive solutes will be preferred; $\mathrm{Na}^{+}$and $\mathrm{Li}^{+}$will be effectively excluded. Two observations implicate additional unknown factors in defining PSAC solute selectivity. First, it is not clear how small ions with intact water shells are excluded by a pore large enough to accommodate bulky organic solutes. Second, studies have found important differences in the transport of closely related organic solutes [15].

Our study also provides an improved, shorter protocol for synchronization of parasite cultures, as often required in basic research studies of transcription or translation stagespecificity. It may also be useful for parasite lines that do not tolerate extended exposure to ambient temperature and $\mathrm{O}_{2}$ levels, as are invariably associated with longer protocols.

Although identification of parasite clag genes as determinants of PSAC activity addressed long-standing debates about whether the channel is host- or pathogen-derived [7, $9,40]$, the structural basis of solute recognition, binding, and transport through this channel remains largely unknown. The CLAG proteins lack conventional transmembrane domains for pore formation; they have also been proposed to serve unrelated roles in erythrocyte invasion or cytoadherence $[47,48]$. Functional studies, such as those presented here, should guide inquiries into the molecular and structural basis of permeation through this unusual channel and important antimalarial drug target.

\section{Abbreviations}

PSAC: Plasmodial surface anion channel

$\mathrm{Gdm}^{+}$: Guanidinium

Gdm-Cl: Guanidinium chloride.

\section{Conflict of Interests}

The authors declare that there is no conflict of interests regarding the publication of this paper.

\section{Acknowledgment}

This research was supported by the Intramural Research Program of the National Institutes of Health, National Institute of Allergy and Infectious Diseases. 


\section{References}

[1] P. A. Tamez, S. Bhattacharjee, C. van Ooij et al., "An erythrocyte vesicle protein exported by the malaria parasite promotes tubovesicular lipid import from the host cell surface," PLoS Pathogens, vol. 4, no. 8, Article ID e1000118, 2008.

[2] J. A. Rowe, A. Claessens, R. A. Corrigan, and M. Arman, "Adhesion of Plasmodium falciparum-infected erythrocytes to human cells: Molecular mechanisms and therapeutic implications," Expert Reviews in Molecular Medicine, vol. 11, article e16, 2009.

[3] H. Ginsburg, M. Krugliak, O. Eidelman, and Z. I. Cabantchik, "New permeability pathways induced in membranes of Plasmodium falciparum infected erythrocytes," Molecular and Biochemical Parasitology, vol. 8, no. 2, pp. 177-190, 1983.

[4] S. A. Desai, S. M. Bezrukov, and J. Zimmerberg, "A voltagedependent channel involved in nutrient uptake by red blood cells infected with the malaria parasite," Nature, vol. 406, no. 6799, pp. 1001-1005, 2000.

[5] A. Alkhalil, J. V. Cohn, M. A. Wagner, J. S. Cabrera, T. Rajapandi, and S. A. Desai, "Plasmodium falciparum likely encodes the principal anion channel on infected human erythrocytes," Blood, vol. 104, no. 13, pp. 4279-4286, 2004.

[6] G. Lisk and S. A. Desai, "The plasmodial surface anion channel is functionally conserved in divergent malaria parasites," Eukaryotic Cell, vol. 4, no. 12, pp. 2153-2159, 2005.

[7] W. Nguitragool, A. A. B. Bokhari, A. D. Pillai et al., "Malaria parasite clag3 genes determine channel-mediated nutrient uptake by infected red blood cells," Cell, vol. 145, no. 5, pp. 665-677, 2011.

[8] P. Sharma, K. Wollenberg, M. Sellers et al., "An epigenetic antimalarial resistance mechanism involving parasite genes linked to nutrient uptake," The Journal of Biological Chemistry, vol. 288, no. 27, pp. 19429-19440, 2013.

[9] S. Mira-Martínez, N. Rovira-Graells, V. M. Crowley, L. M. Altenhofen, M. Llinás, and A. Cortés, "Epigenetic switches in clag3 genes mediate blasticidin S resistance in malaria parasites," Cellular Microbiology, vol. 15, pp. 1913-1923, 2013.

[10] A. D. Pillai, W. Nguitragool, B. Lyko et al., "Solute restriction reveals an essential role for clag3-associated channels in malaria parasite nutrient acquisition," Molecular Pharmacology, vol. 82, no. 6, pp. 1104-1114, 2012.

[11] H. Ginsburg, S. Kutner, M. Krugliak, and Z. I. Cabantchik, "Characterization of permeation pathways appearing in the host membrane of Plasmodium falciparum infected red blood cells," Molecular and Biochemical Parasitology, vol. 14, no. 3, pp. 313-322, 1985.

[12] A. M. Gero and A. M. Wood, "New nucleoside transport pathways induced in the host erythrocyte membrane of malaria and babesia infected cells," Advances in Experimental Medicine and Biology, vol. 309, pp. 169-172, 1991.

[13] K. J. Saliba, H. A. Horner, and K. Kirk, “Transport and metabolism of the essential vitamin pantothenic acid in human erythrocytes infected with the malaria parasite Plasmodium falciparum," The Journal of Biological Chemistry, vol. 273, no. 17, pp. 10190-10195, 1998.

[14] H. M. Staines, C. Rae, and K. Kirk, "Increased permeability of the malaria-infected erythrocyte to organic cations," Biochimica et Biophysica Acta, vol. 1463, no. 1, pp. 88-98, 2000.

[15] A. A. B. Bokhari, T. Solomon, and S. A. Desai, "Two distinct mechanisms of transport through the plasmodial surface anion channel," Journal of Membrane Biology, vol. 226, no. 1-3, pp. 2734,2008 .

[16] J. V. Cohn, A. Alkhalil, M. A. Wagner, T. Rajapandi, and S. A. Desai, "Extracellular lysines on the plasmodial surface anion channel involved in $\mathrm{Na}^{+}$exclusion," Molecular and Biochemical Parasitology, vol. 132, no. 1, pp. 27-34, 2003.

[17] A. D. Pillai, R. Addo, P. Sharma, W. Nguitragool, P. Srinivasan, and S. A. Desai, "Malaria parasites tolerate a broad range of ionic environments and do not require host cation remodelling," Molecular Microbiology, vol. 88, no. 1, pp. 20-34, 2013.

[18] P. Lee, Z. Ye, K. Van Dyke, and R. G. Kirk, "X-ray microanalysis of Plasmodium falciparum and infected red blood cells: effects of qinghaosu and chloroquine on potassium, sodium, and phosphorus composition," American Journal of Tropical Medicine and Hygiene, vol. 39, no. 2, pp. 157-165, 1988.

[19] J. M. A. Mauritz, R. Seear, A. Esposito et al., "X-ray microanalysis investigation of the changes in $\mathrm{Na}, \mathrm{K}$, and hemoglobin concentration in Plasmodium falciparum-infected red blood cells," Biophysical Journal, vol. 100, no. 6, pp. 1438-1445, 2011.

[20] B. Hille, Ion Channels of Excitable Membranes, Medicine \& Health Science Books, 3rd edition, 2001.

[21] M. A. Wagner, B. Andemariam, and S. A. Desai, "A two-compartment model of osmotic lysis in Plasmodium falciparuminfected erythrocytes," Biophysical Journal, vol. 84, no. 1, pp. 116123, 2003.

[22] D. A. Hill and S. A. Desai, "Malaria parasite mutants with altered eryth rocyte permeability: a new drug resistance mechanism and important molecular tool," Future Microbiology, vol. 5, no. 1, pp. 81-97, 2010.

[23] G. Lisk, M. Pain, I. Y. Gluzman et al., "Changes in the plasmodial surface anion channel reduce leupeptin uptake and can confer drug resistance in Plasmodium falciparum-infected erythrocytes," Antimicrobial Agents and Chemotherapy, vol. 52, no. 7, pp. 2346-2354, 2008.

[24] G. Lisk, M. Pain, M. Sellers et al., "Altered plasmodial surface anion channel activity and in vitro resistance to permeating antimalarial compounds," Biochimica et Biophysica Acta, vol. 1798, no. 9, pp. 1679-1688, 2010.

[25] S. Sokolov, T. Scheuer, and W. A. Catterall, "Ion permeation and block of the gating pore in the voltage sensor of $\mathrm{Na}_{v} 1.4$ channels with hypokalemic periodic paralysis mutations," Journal of General Physiology, vol. 136, no. 2, pp. 225-236, 2010.

[26] A. D. Pillai, M. Pain, T. Solomon, A. A. B. Bokhari, and S. A. Desai, "A cell-based high-throughput screen validates the plasmodial surface anion channel as an antimalarial target," Molecular Pharmacology, vol. 77, no. 5, pp. 724-733, 2010.

[27] A. Alkhalil, A. D. Pillai, A. A. B. Bokhari, A. B. Vaidya, and S. A. Desai, "Complex inheritance of the plasmodial surface anion channel in a Plasmodium falciparum genetic cross," Molecular Microbiology, vol. 72, no. 2, pp. 459-469, 2009.

[28] G. Lisk, S. Scott, T. Solomon, A. D. Pillai, and S. A. Desai, "Solute-inhibitor interactions in the plasmodial surface anion channel reveal complexities in the transport process," Molecular Pharmacology, vol. 71, no. 5, pp. 1241-1250, 2007.

[29] W. Nguitragool, K. Rayavara, and S. A. Desai, "Proteolysis at a specific extracellular residue implicates integral membrane CLAG3 in malaria parasite nutrient channels," PLOS ONE, vol. 9, no. 4, p. e93759, 2014.

[30] C. Lambros and J. P. Vanderberg, "Synchronization of Plasmodium falciparum erythrocytic stages in culture," Journal of Parasitology, vol. 65, no. 3, pp. 418-420, 1979. 
[31] S. Kutner, W. V. Breuer, H. Ginsburg, S. B. Aley, and Z. I. Cabantchik, "Characterization of permeation pathways in the plasma membrane of human erythrocytes infected with early stages of Plasmodium falciparum: association with parasite development," Journal of Cellular Physiology, vol. 125, no. 3, pp. 521-527, 1985.

[32] I. D. Goodyer, J. Johnson, R. Eisenthal, and D. J. Hayes, "Purification of mature-stage Plasmodium falciparum by gelatine flotation," Annals of Tropical Medicine and Parasitology, vol. 88, no. 2, pp. 209-211, 1994.

[33] T. Staalsoe, H. A. Giha, D. Dodoo, T. G. Theander, and L. Hviid, "Detection of antibodies to variant antigens on Plasmodium falciparum-infected erythrocytes by flow cytometry," Cytometry, vol. 35 , no. 4, pp. 329-336, 1999.

[34] D. Lerche and R. Glaser, "Investigations of artificial aggregation of washed human erythrocytes caused by decreased $\mathrm{pH}$ and reduced ionic strength," Acta Biologica et Medica Germanica, vol. 39, no. 8-9, pp. 973-978, 1980.

[35] D. A. Hill, A. D. Pillai, F. Nawaz et al., "A blasticidin S-resistant Plasmodium falciparum mutant with a defective plasmodial surface anion channel," Proceedings of the National Academy of Sciences of the United States of America, vol. 104, no. 3, pp. 10631068, 2007.

[36] R. R. Overman, "Reversible cellular permeability alterations in disease. In vivo studies on sodium, potassium and chloride concentrations in erythrocytes of the malarious monkey," American Journal of Physiology, vol. 152, pp. 113-121, 1948.

[37] C. A. Homewood and K. D. Neame, "Malaria and the permeability of the host erythrocyte," Nature, vol. 252, no. 5485, pp. 718-719, 1974.

[38] J. M. Upston and A. M. Gero, "Parasite-induced permeation of nucleosides in Plasmodium falciparum malaria," Biochimica et Biophysica Acta, vol. 1236, no. 2, pp. 249-258, 1995.

[39] S. A. Desai, "Why do malaria parasites increase host erythrocyte permeability?" Trends in Parasitology, vol. 30, no. 3, pp. 151-159, 2014.

[40] H. M. Staines, A. Alkhalil, R. J. Allen et al., "Electrophysiological studies of malaria parasite-infected erythrocytes: current status," International Journal for Parasitology, vol. 37, no. 5, pp. 475-482, 2007.

[41] V. L. Lew and R. M. Bookchin, "Volume, pH, and ion-content regulation in human red cells: analysis of transient behavior with an integrated model," Journal of Membrane Biology, vol. 92, no. 1, pp. 57-74, 1986.

[42] G. Eisenman and R. Horn, "Ionic selectivity revisited: the role of kinetic and equilibrium processes in ion permeation through channels," Journal of Membrane Biology, vol. 76, no. 3, pp. 197$225,1983$.

[43] E. M. Wright and J. M. Diamond, "Anion selectivity in biological systems," Physiological Reviews, vol. 57, no. 1, pp. 109-156, 1977.

[44] P. E. Mason, G. W. Neilson, C. E. Dempsey, A. C. Barnes, and J. M. Cruickshank, "The hydration structure of guanidinium and thiocyanate ions: implications for protein stability in aqueous solution," Proceedings of the National Academy of Sciences of the United States of America, vol. 100, no. 8, pp. 4557-4561, 2003.

[45] Y. Zhang and P. S. Cremer, "Interactions between macromolecules and ions: the Hofmeister series," Current Opinion in Chemical Biology, vol. 10, no. 6, pp. 658-663, 2006.

[46] C. M. Armstrong, "Reflections on selectivity," in Membrane Transport, pp. 261-273, Springer, Berlin, Germany, 1989.
[47] K. R. Trenholme, D. L. Gardiner, D. C. Holt, E. A. Thomas, A. F. Cowman, and D. J. Kemp, "clag9: a cytoadherence gene in Plasmodium falciparum essential for binding of parasitized erythrocytes to CD36," Proceedings of the National Academy of Sciences of the United States of America, vol. 97, no. 8, pp. 40294033, 2000.

[48] O. Kaneko, "Erythrocyte invasion: vocabulary and grammar of the Plasmodium rhoptry," Parasitology International, vol. 56, no. 4, pp. 255-262, 2007. 

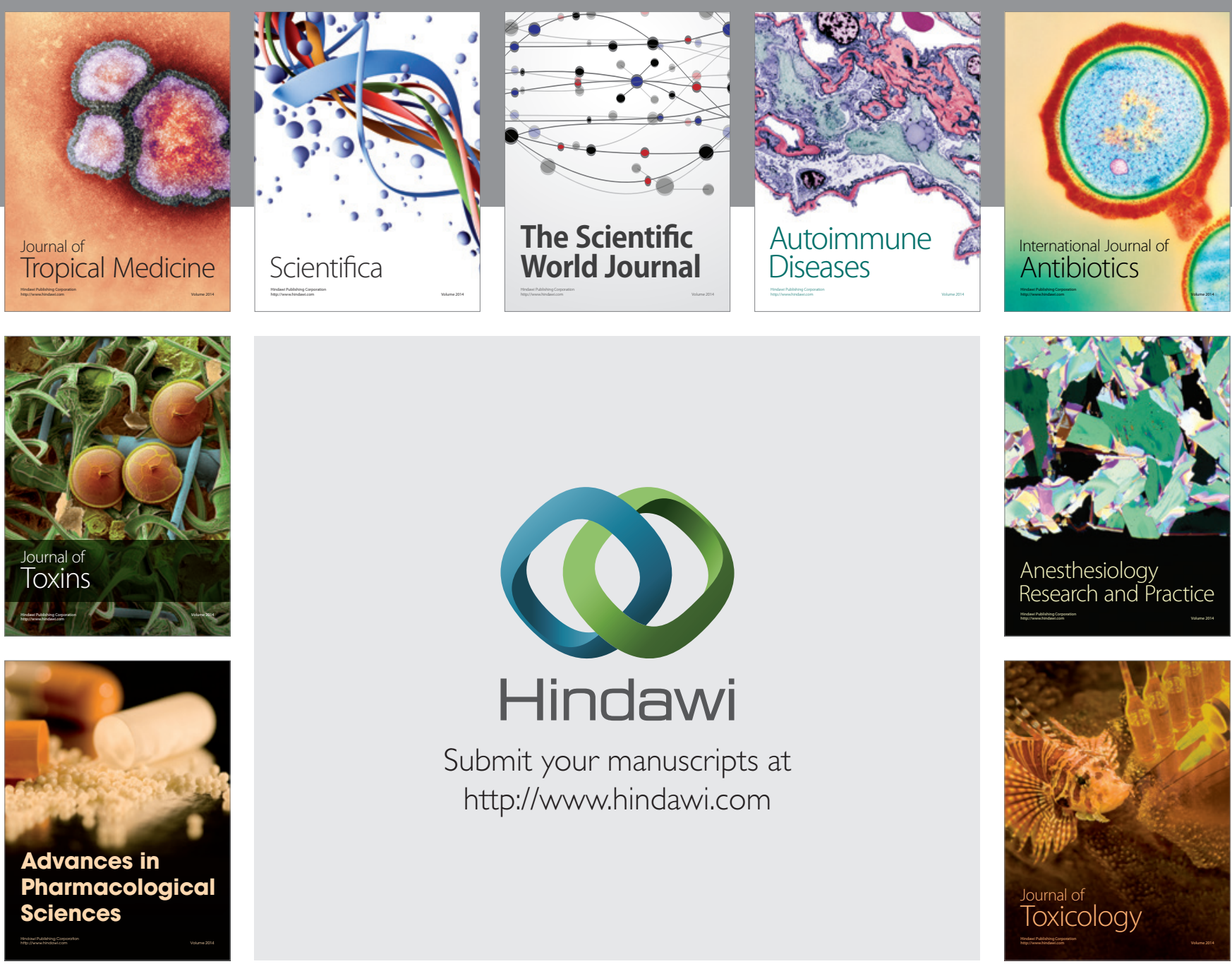

\section{Hindawi}

Submit your manuscripts at

http://www.hindawi.com
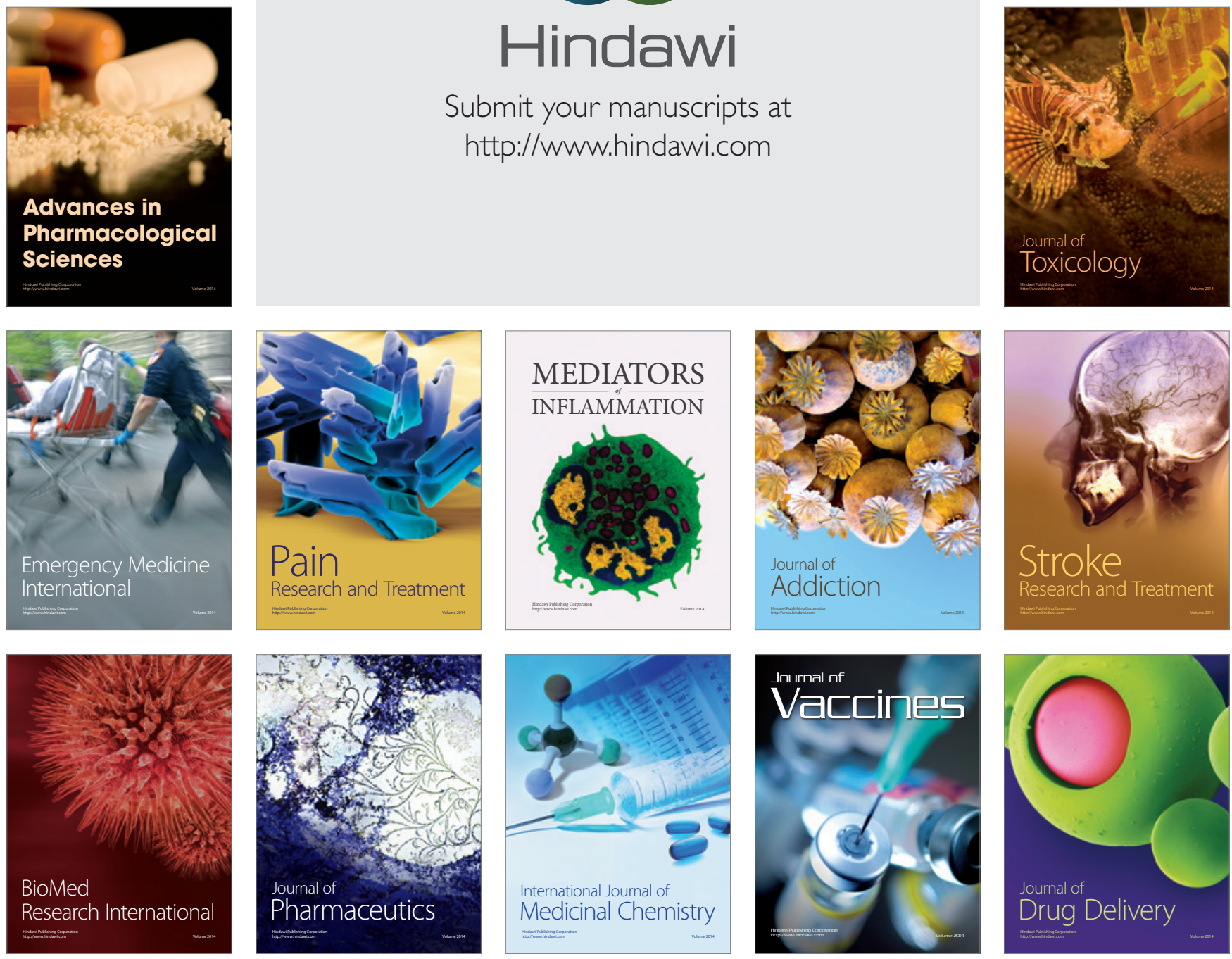